\title{
Cryomodule tests of four Tesla-like cavities in the Superconducting RF Test Facility at KEK
}

\author{
Eiji Kako, ${ }^{*}$ Hitoshi Hayano, Shuichi Noguchi, Norihito Ohuchi, Masato Satoh, Toshio Shishido, \\ Ken Watanabe, and Yasuchika Yamamoto \\ KEK, High Energy Accelerator Research Organisation 1-1, Oho, Tsukuba, Ibaraki, 305-0801, Japan
}

(Received 29 November 2009; published 29 April 2010)

\begin{abstract}
A 6-m cryomodule including four Tesla-like cavities was developed, and was tested in the Superconducting RF Test Facility phase-I at KEK. The performance as a total superconducting cavity system was checked in the cryomodule tests at $2 \mathrm{~K}$ with high rf power. One of the four cavities achieved a stable pulsed operation at $32 \mathrm{MV} / \mathrm{m}$, which is higher than the operating accelerating gradient in the ILC. The maximum accelerating gradient $\left(E_{\text {acc, max }}\right)$ obtained in the vertical cw tests was maintained or slightly improved in the cryomodule tests operating in a pulse mode. Compensation of the Lorentz force detuning at $31 \mathrm{MV} / \mathrm{m}$ was successfully demonstrated by a piezo tuner and predetuning.
\end{abstract}

DOI: 10.1103/PhysRevSTAB.13.041002

PACS numbers: 29.20.Ej

\section{INTRODUCTION}

The construction of a Superconducting RF Test Facility (STF) at KEK has been ongoing since 2005, for the future International Linear Collider (ILC) project. The main purpose of the STF is to develop cryomodules containing high gradient cavities, which can be stably operated at an average accelerating gradient of $31.5 \mathrm{MV} / \mathrm{m}$. A superconducting cavity system, which includes four Tesla-like nine-cell cavities, input couplers, and frequency tuners, was designed and fabricated [1]. Component tests of the ninecell cavities and the input couplers were carried out in order to qualify their performances [2]. In the first step, called the STF phase- 0.5 , a 6-m cryomodule containing one Tesla-like cavity had been tested in 2007. Overall, the superconducting cavity system worked well, without any major problems, in the initial test [3,4]. In the second step, called the STF phase-I, the assembly of the 6-m cryomodule containing four Tesla-like cavities was started in January 2008. After the cryomodule was installed in the tunnel and cooled, high power tests at $2 \mathrm{~K}$ were performed from July to December 2008. The main purposes of these cryomodule tests in the STF phase-I are summarized as follows: (i) to check the performance as a total superconducting cavity system and find out the components for improvement for the future project; (ii) to confirm a stable pulsed operation at higher accelerating gradients and compare the $E_{\text {acc, max }}$ achieved in the cryomodule tests with the results from vertical cw tests; and (iii) to demonstrate the compensation of the Lorentz force detuning by a piezo tuner and establish the effectiveness of an improved stiffness in the cavity support structure.

The components of the cavity package in a Tesla-like cavity system and the assembly procedure for the 6-m cryomodule are briefly reported in the following sections. The high gradient performance of the four Tesla-like cav-

*eiji.kako@kek.jp ities in the cryomodule tests is mainly described in this paper.

\section{COMPONENTS IN CAVITY PACKAGE}

A superconducting cavity system consists of a nine-cell niobium cavity with titanium end plates and two higherorder mode (HOM) couplers, an input coupler with cold and warm rf windows, a mechanical frequency tuner with a piezo element, a helium vessel made of titanium, and a magnetic shield.

\section{A. Tesla-like nine-cell cavity}

The stiffness of the cavity support structure is very important to suppress cell shrinkage in the axial direction due to the Lorentz force in a pulsed operation. As shown in Fig. 1, the Tesla-like nine-cell cavity developed at KEK has thick titanium end plates, in order to improve the stiffness of the Tesla cavity designed at DESY [5]. Therefore, a specific feature of the Tesla-like cavities is the improved stiffness of the total cavity support system, which consists of end plates, a cylinder, and a tuning mechanism. A cavity structure comparison between the Tesla cavity at DESY and the Tesla-like cavity at KEK is shown Fig. 2. The main cavity parameters are listed in Table I. The modifications added to the Tesla-like cavities are summarized as follows: (i) improved support stiffness by thick titanium end plates; (ii) enlarged beam pipe diameter to compensate for the weakened input coupling

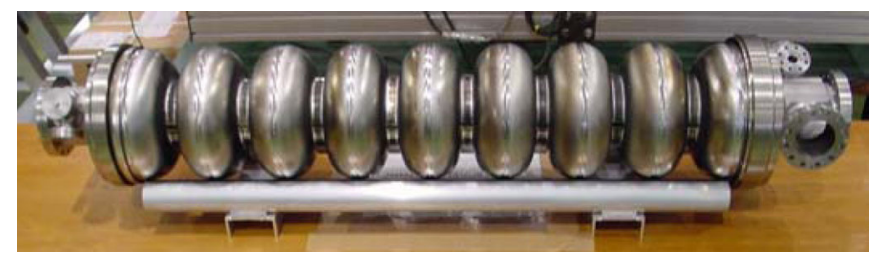

FIG. 1. (Color) Tesla-like nine-cell niobium cavity developed at KEK. 


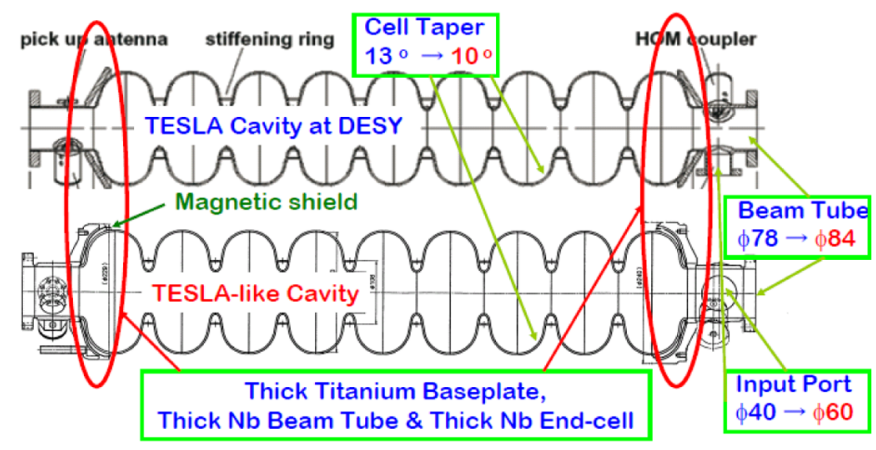

FIG. 2. (Color) Comparison between cavity structures of Tesla cavity at DESY (top) and Tesla-like cavity at KEK (bottom). due to the thick end plates; (iii) enlarged input port diameter to make the usable rf window larger and increase the power capability; (iv) reoptimization of cell shape to decrease the ratio of the surface peak magnetic field $\left(H_{\mathrm{sp}}\right)$ to the accelerating field $\left(E_{\text {acc }}\right)$; $(\mathrm{v})$ overcoupling of an input coupler to widen the bandwidth and ensure more stable operation; and (vi) installation of a magnetic shield inside the helium vessel.

As a result of the improved cavity support system, the stiffness was increased from 22 to $72 \mathrm{kN} / \mathrm{mm}$ in the calculated values. Therefore, suppression of the Lorentzdetuning frequency from -500 to $-150 \mathrm{~Hz}$ is expected during operation at $31.5 \mathrm{MV} / \mathrm{m}$. The confirmation of this

TABLE I. Comparison of main cavity parameters.

\begin{tabular}{lcc}
\hline \hline Cavity & Tesla/DESY & Tesla-like/KEK \\
\hline Cell taper & $76.7^{\circ}$ & $80.0^{\circ}$ \\
Diameter of beam tube & $78 \mathrm{~mm}$ & $84 \mathrm{~mm}$ \\
Diameter of input port & $40 \mathrm{~mm}$ & $60 \mathrm{~mm}$ \\
$E_{\mathrm{sp}} / E_{\mathrm{acc}}$ & 1.98 & 2.17 \\
$H_{\mathrm{sp}} / E_{\mathrm{acc}}$ & $42.6 \mathrm{Oe} / \mathrm{MV} / \mathrm{m}$ & $41.0 \mathrm{Oe} / \mathrm{MV} / \mathrm{m}$ \\
$R / Q$ & $1036 \Omega$ & $1016 \Omega$ \\
Input coupling & $3.0 \times 10^{6}$ & $2.0 \times 10^{6}$ \\
Stiffness of support system & $22 \mathrm{kN} / \mathrm{mm}$ & $72 \mathrm{kN} / \mathrm{mm}$ \\
Estimated Lorentz detuning at $31.5 \mathrm{MV} / \mathrm{m}$ & $-500 \mathrm{~Hz}$ in a flattop & $-150 \mathrm{~Hz}$ in a flattop \\
\hline \hline
\end{tabular}

TABLE II. Main specifications of input couplers.

\begin{tabular}{ll}
\hline \hline Frequency & $1.3 \mathrm{GHz}$ \\
Pulse width & $1.5 \mathrm{~ms}$ \\
Repetition rate & $5 \mathrm{~Hz}$ \\
Beam current & $9 \mathrm{~mA}$ \\
Accelerating gradient & $31.5 \mathrm{MV} / \mathrm{m}$ \\
rf peak power & $300 \mathrm{~kW}$ \\
External $Q$ value & $2.0 \times 10^{6}$ \\
Half bandwidth & $325 \mathrm{~Hz}$ \\
Over coupling ratio & 1.5 \\
\hline \hline
\end{tabular}
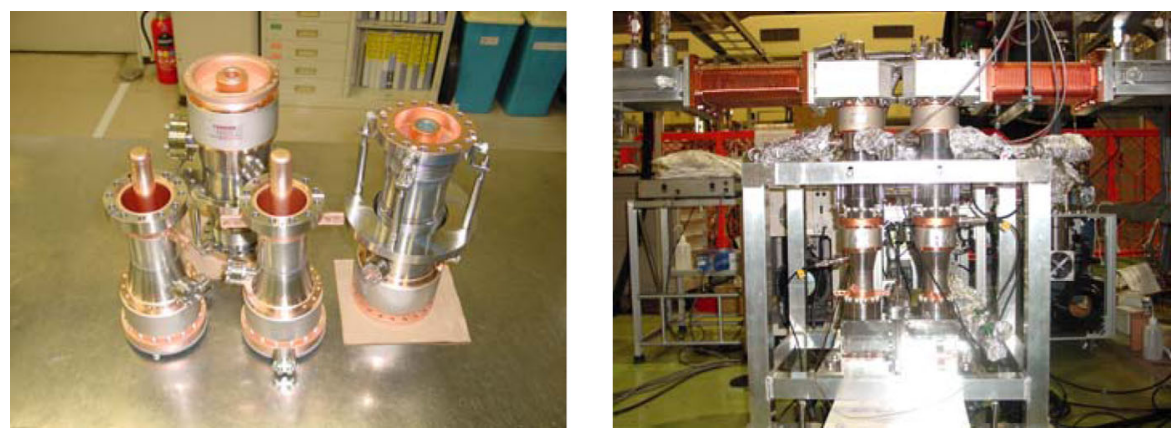

FIG. 3. (Color) Two pairs of input couplers with cold and warm rf windows (left) and setup of high power test stand (right). 
improved effect was the most interesting experiment in the high power tests of the 6-m cryomodule.

\section{B. Input couplers}

Main specifications of input couplers are listed in Table II. The input coupler, which consists of a cold and

TABLE III. Calculated static and dynamic thermal losses of input coupler. (The outer conductor at the cold part consists of 1$\mathrm{mm}$ thick stainless steel and 5- $\mu \mathrm{m}$ thick copper plating.)

\begin{tabular}{llll}
\hline \hline & $80 \mathrm{~K}$ & $5 \mathrm{~K}$ & $2 \mathrm{~K}$ \\
\hline Static loss [W] & 5.0 & 1.1 & 0.05 \\
Dynamic loss [W] & 3.0 & 0.2 & 0.03 \\
\hline \hline
\end{tabular}

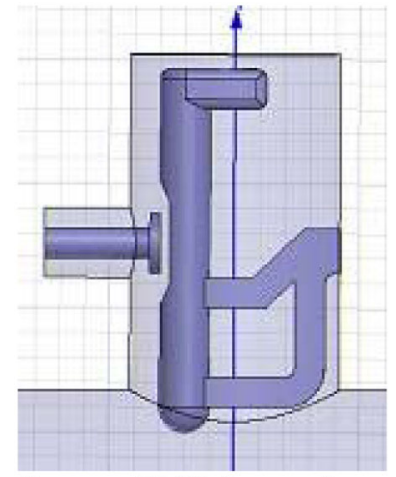

L-type HOM coupler

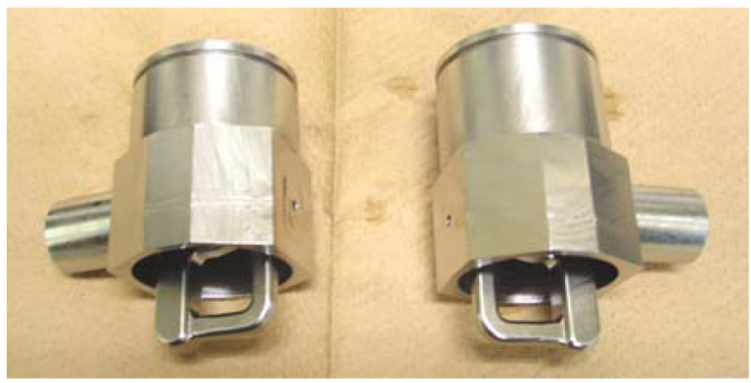

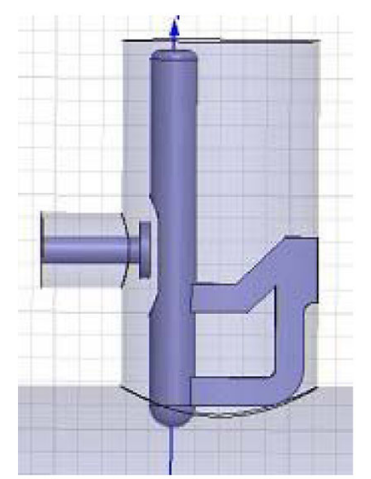

I-type HOM coupler
FIG. 4. (Color) Two types of HOM couplers.

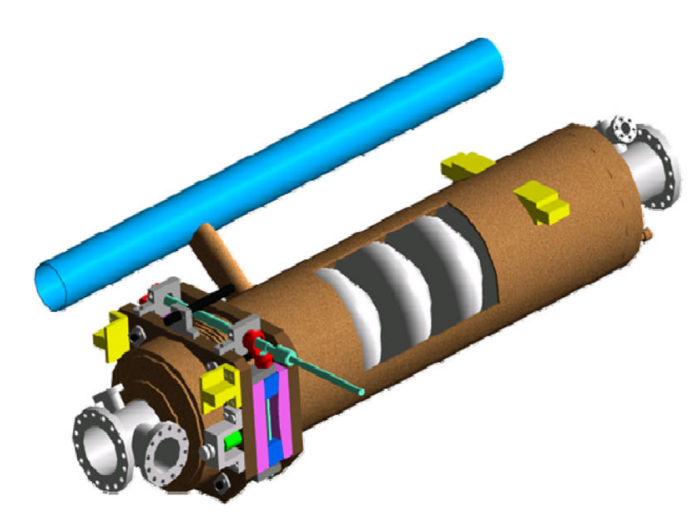

a warm coupler, has two TRSTAN-type coaxial disk rf windows [6], as shown at the left in Fig. 3. There is no tuning mechanism for varying the coupling for the sake of simplicity and cost reduction. In particular, a bellows at the outer conductor in the $5 \mathrm{~K}$ part was eliminated in order to make the cavity assembly work easier. Four input couplers were fabricated, and high power tests were carried out in a test stand with a 5-MW pulsed klystron, as shown at the right in Fig. 3.

The calculated static and dynamic thermal losses at an input coupler are summarized in Table III. The static loss of $1.1 \mathrm{~W}$ at $5 \mathrm{~K}$ is 5 times larger than the dynamic loss of $0.2 \mathrm{~W}$ at an input rf power of $350 \mathrm{~kW}$. The elimination of the thin bellows at the outer conductor in the $5 \mathrm{~K}$ part might be one of the causes of the high static loss at $5 \mathrm{~K}$.

\section{HOM couplers}

Higher order mode (HOM) couplers are located in both beam tubes of the cavities, as shown in Figs. 1 and 2. Two types of HOM couplers (L-type and I-type) [7], which were based on the Tesla design at DESY [5], were fabricated, as shown in Fig. 4. Their filter characteristics were optimized to give them different 2 nd stop-band frequencies.

\section{Frequency tuner}

A slide-jack tuner system with the piezo element shown in Fig. 5 was newly developed for the Tesla-like cavity at KEK. The frequency tuning system consists of a slide-jack tuner for mechanical slow tuning and a piezo tuner for electrical fast tuning to compensate for the Lorentz force detuning during an $\mathrm{rf}$ pulse. The stepping motor is mounted outside the cryomodule, making it very easy to replace the motor when a problem occurs. Likewise, the piezo element is attached at a location that allows it to be replaced without disassembling the cryomodule.

Main specifications of the slide-jack tuner system consisting of a coarse tuner (stepping motor) and a fine tuner (piezo) are listed in Table IV.

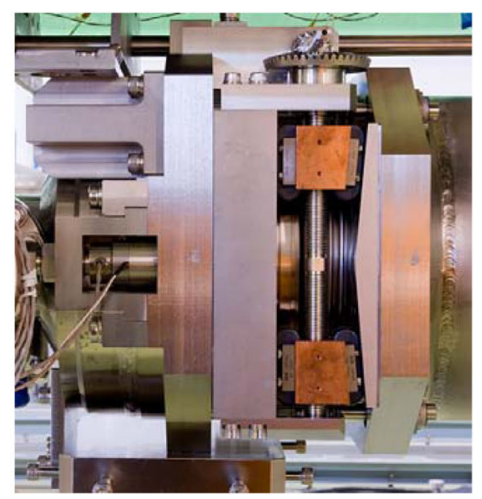

FIG. 5. (Color) Slide-jack tuner system for adjusting resonant frequency. A schematic drawing of the entire cavity system (left) and the completed assembly of the frequency tuner system with a pair of slide jacks and a piezo element (right). 
TABLE IV. Main specifications of frequency tuning system.

\begin{tabular}{lll}
\hline \hline Coarse tuner & Slide-jack driven by a stepping motor \\
\hline & Sliding wedge & 2 \\
& Stroke & $<4 \mathrm{~mm}(1.2 \mathrm{MHz})$ \\
& Resolution & $<0.1 \mu \mathrm{m}(30 \mathrm{~Hz})$ \\
\hline Fine tuner & Piezo stack element & \\
\hline & Voltage & $1.0 \mathrm{kV}$ type for one cavity \\
& $150 \mathrm{~V}$ type for three cavities \\
& Stroke & $>4 \mu \mathrm{m}$ at $2 \mathrm{~K}(600 \mathrm{~Hz})$ \\
& Resolution & $<0.01 \mu \mathrm{m}(1.5 \mathrm{~Hz})$ \\
& Maximum load & $3.5 \times 10^{4} \mathrm{~N}$ \\
& Stiffness & $4.0 \times 10^{5} \mathrm{~N} / \mathrm{mm}$ \\
\hline \hline
\end{tabular}
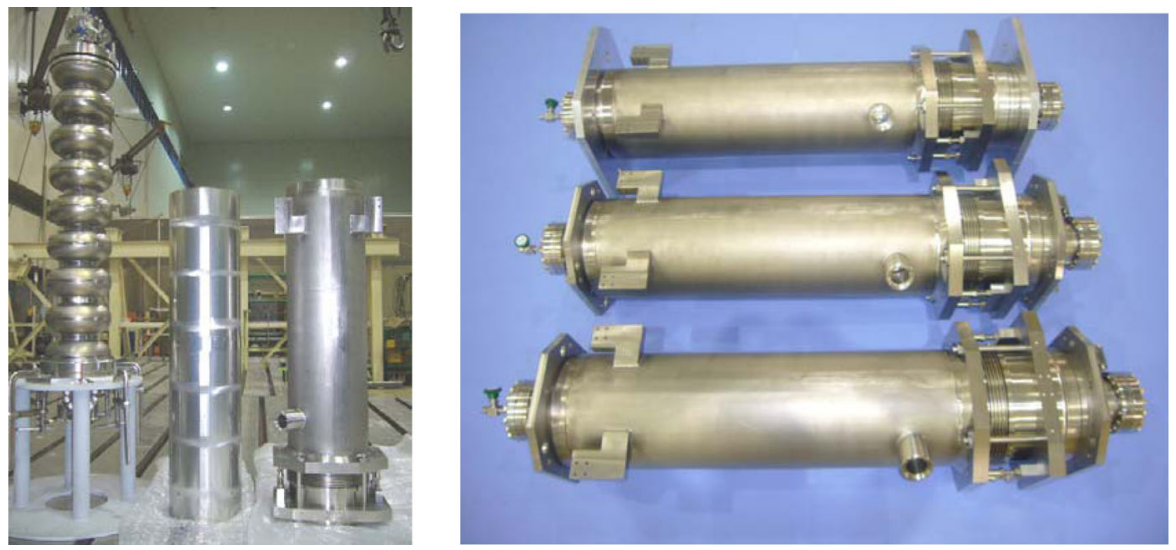

FIG. 6. (Color) Tesla-like nine-cell cavity, magnetic shield, and titanium helium vessel (left). Three completed cavities after welding of helium jacket (right).

\section{E. Helium vessel}

After the qualification of the cavity performance in the vertical tests [8], the four cavities were transported to a welding company. The Tesla-like nine-cell cavities were covered with a magnetic shield and a helium jacket designed to be filled with liquid helium, as shown in Fig. 6 (left). The cavity end plates were joined to the titanium helium vessel by tungsten inert gas welding, as shown in Fig. 6 (right).

\section{CRYOMODULE ASSEMBLY AND COUPLER CONDITIONING}

\section{A. Assembly procedure for cryomodule}

The assembly procedure for the 6-m cryomodule containing four Tesla-like cavities is summarized as follows: (i) The string assembly of the four cavities was performed in a class-10 clean room. This included the attachment of input couplers with a cold rf window and HOM pickup antennas, connection of a bellows duct between two cavities, and installation of a gate-valve in each end of the beam-tube (Fig. 7). (ii) In a class-1000 clean room, after a vacuum leak-check of the string cavities, argon gas was

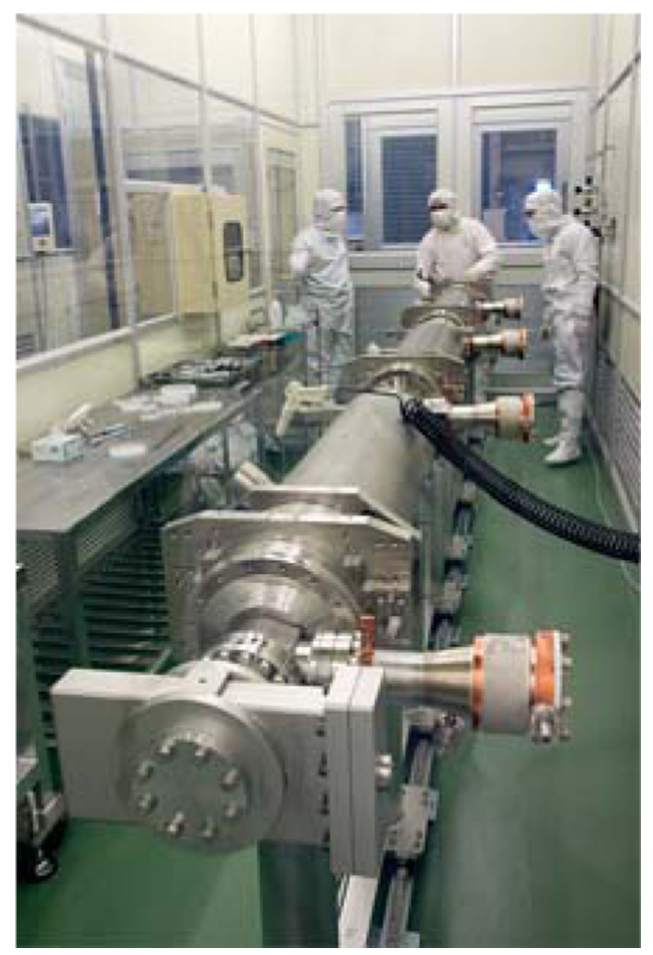

FIG. 7. (Color) Cavity string assembly in class-10 clean room. 


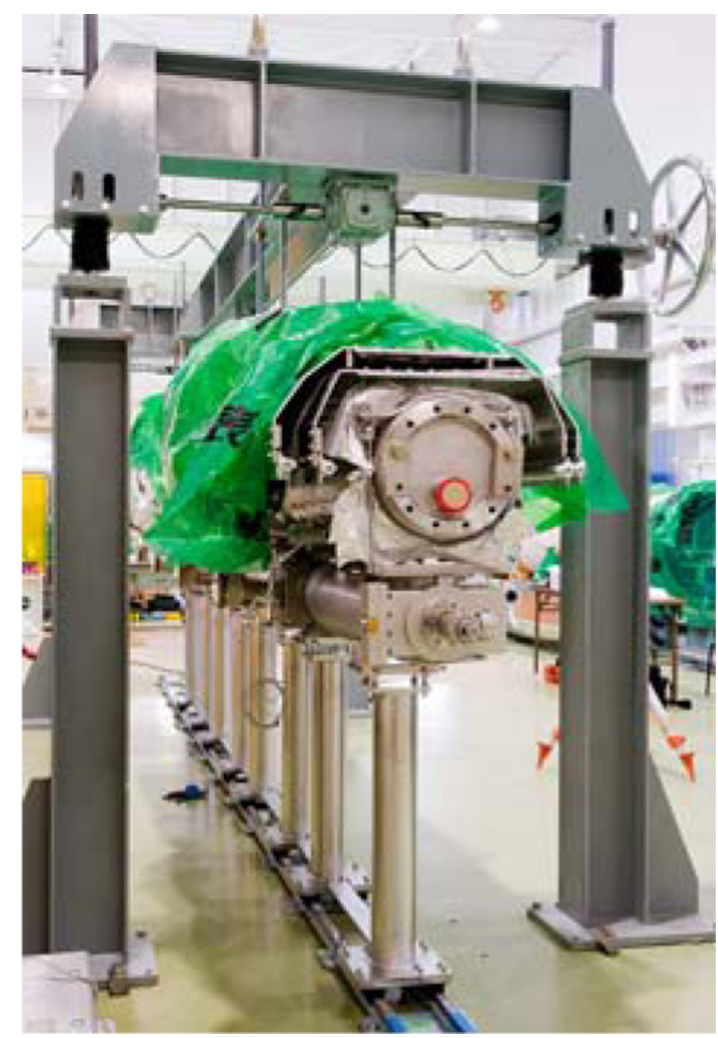

FIG. 8. (Color) Hanging string cavities on a GRP.

introduced very slowly for $\sim 40 \mathrm{~h}$ up to atmospheric pressure. (iii) The attachment of a frequency tuning system and alignment of the four cavities were carried out in an area outside the clean room. (iv) In a cryomodule assembly area, the string cavities were hung on a helium gas return pipe (GRP), as shown in Fig. 8, and the cavities were covered with $5 \mathrm{~K}$ and $80 \mathrm{~K}$ thermal shields. (v) An assembled cold mass, including the four Tesla-like nine-cell cavities, was inserted into a vacuum vessel, as shown at the left in Fig. 9. (vi) The completed 6-m cryomodule was installed in the STF tunnel. The cryomodule was connected to a cold box that supplied liquid helium and liquid nitrogen. The input couplers of the four cavities were connected to a high power rf source through an rf power distribution system [9] consisting of waveguides, circulators, power dividers, and directional couplers, as shown at the right in Fig. 9.

\section{B. Conditioning of input couplers}

Prior to the assembly of the nine-cell cavities (Fig. 7), the input couplers were conditioned using a high power test stand, as shown at the right in Fig. 3. The rf input power required for the conditioning in the traveling wave mode at the test stand was determined to be 4 times higher than that in the standing wave mode in the cryomodule. The conditioning was initially started using short pulses of 0.01 or $0.1 \mathrm{~ms}$, and rf power level was increased very carefully. Finally, conditioning up to $1.0 \mathrm{MW}$ in a pulsed operation with $1.5 \mathrm{~ms}$ pulse width at $5 \mathrm{~Hz}$ was successfully performed on the four input couplers. The conditioning time at the test stand was approximately $50 \mathrm{~h}$, as shown in Fig. 10.

After the installation of the cryomodule in the STF tunnel, the connection of a warm coupler with a cold coupler was carried out in a work area covered with a special clean booth to maintain a clean environment. In situ baking of cold rf windows inside the cryomodule was carried out at $85^{\circ} \mathrm{C}$ for $15 \mathrm{~h}$. The baking of cold rf windows prior to conditioning is very effective at reducing the conditioning time. The conditioning of the input couplers at room temperature before cooling was carried out at up to 240-330 kW under a total reflection condition. The conditioning time was $11-17 \mathrm{~h}$, as shown in Fig. 11. Processing of the No. 3 coupler (C/\#2 cavity) was carried out twice, and the second processing time was remarkably short. It is considered that a memory of the previous processing was preserved.

\section{LOW POWER RF MEASUREMENT}

\section{A. Slide-jack tuner}

The tuning characteristics of the four cavities in low power $\mathrm{rf}$ measurements at $2 \mathrm{~K}$ are shown in Fig. 12. The dynamic range of the resonant frequency change was $600-800 \mathrm{kHz}$, and the frequency sensitivity was approximately $300 \mathrm{kHz}$ per mm, as expected. However, one cavity
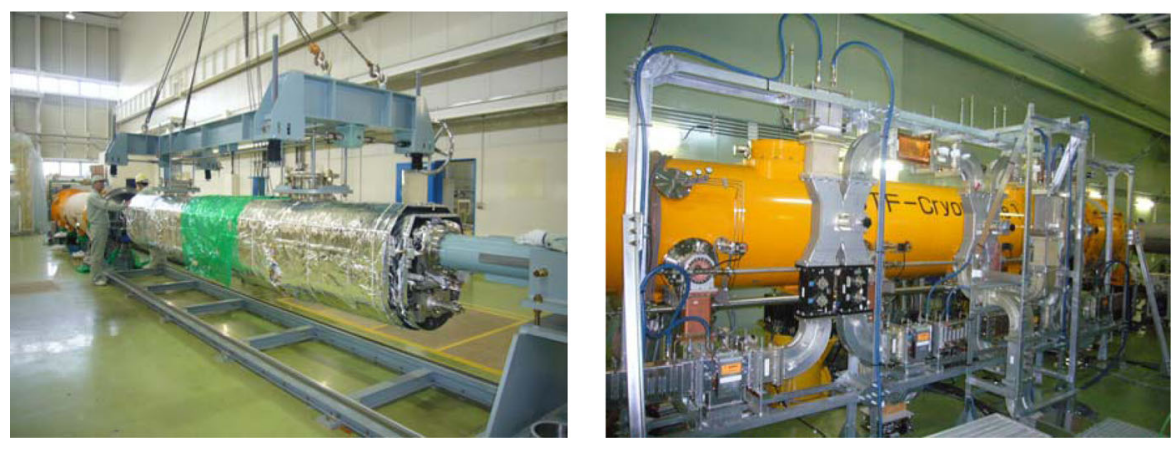

FIG. 9. (Color) Insertion of assembled cold mass into vacuum vessel (left) and installation of cryomodule in STF tunnel (right). 


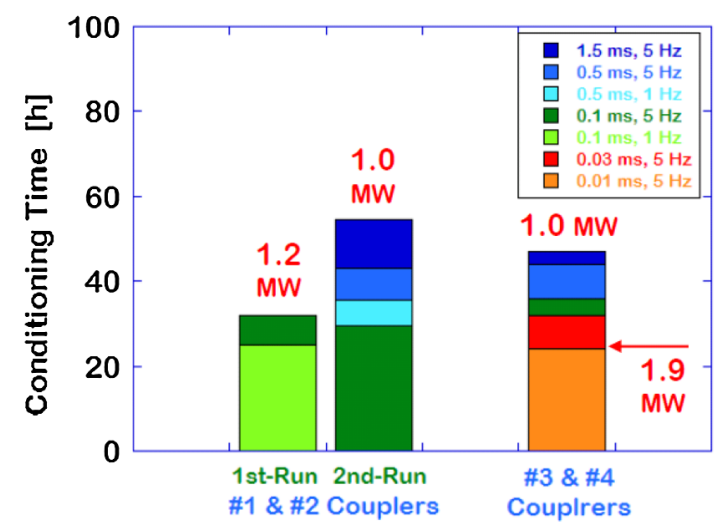

FIG. 10. (Color) Conditioning time for two pairs of input couplers at test stand under matching condition (traveling wave mode).

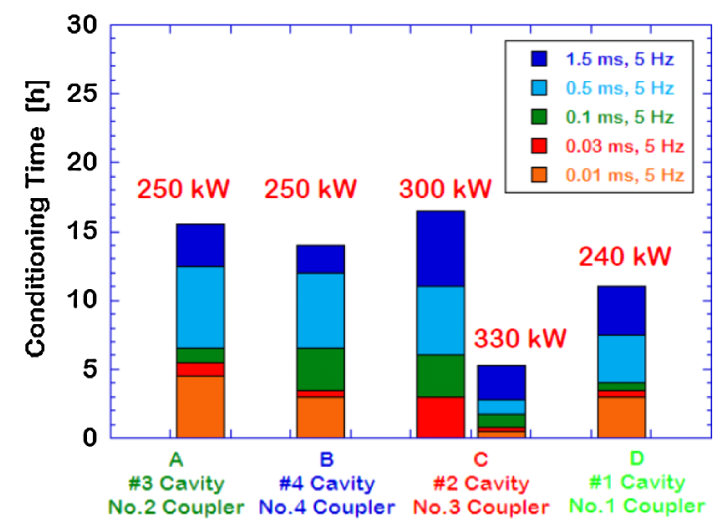

FIG. 11. (Color) Conditioning time for four input couplers in cryomodule under total reflection condition (standing wave mode).

(A/\#3) showed a strange performance, which might be due to a mistake in adjusting the true frequency during the tuning process at room temperature. Therefore, the operating rf frequency was changed from 1300.000 to $1300.500 \mathrm{MHz}$ in order to simultaneously drive the four cavities, as shown with the orange lines in Fig. 12. The initial stepping motors (1-Nm type) were replaced with new ones of the 5-Nm type, because the torque to rotate a drive shaft was insufficient at low temperature. Because the stepping motors were located outside the cryomodule, it was easy to exchange them.

\section{B. Piezo tuner}

The optimization of the timing, frequency, amplitude, and waveform of the drive pulse signal to a piezo tuner is important for an effective compensation of Lorentz force detuning. A maximum load of $5 \mathrm{kN}$ and a stroke at low temperature of approximately $4 \mu \mathrm{m}$ are required. Three low-voltage-type (maximum $+150 \mathrm{~V}$ ) and one high-voltage-type (maximum $+1000 \mathrm{~V}$ ) piezo elements were used

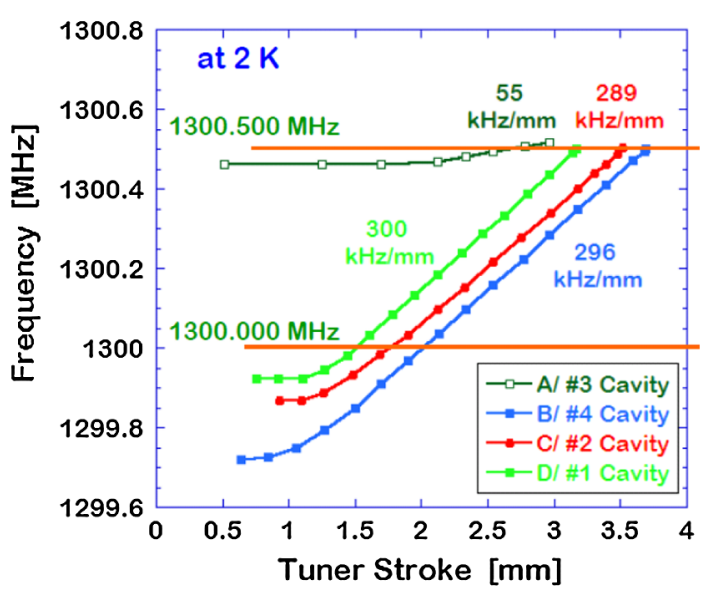

FIG. 12. (Color) Cavity resonant frequency vs tuner stroke equivalent to changing a total cavity length driven by the slide-jack tuner.

in the piezo tuners for the four cavities, in order to compare their performances. The measurement of the single pulse response driven by the piezo tuner is shown in Fig. 13 (left), and the delay time from the input pulse signal to the change in the cavity phase $(\phi)$ was approximately $0.7 \mathrm{~ms}$. The results of the frequency change $(\Delta f)$ estimated by the phase shift $(\Delta \phi)$ are shown in Fig. 13 (right). A frequency change of more than $300 \mathrm{~Hz}$ was obtained in the high-voltage-type piezo $(+500 \mathrm{~V})$. On the other hand, the frequency change in the low-voltage-type piezo $(+100 \mathrm{~V})$ was considerably lower than the expected Lorentz-detuning frequency at $31.5 \mathrm{MV} / \mathrm{m}$. The improvement of the piezo tuner system with the low-voltage type is needed for the next step.

A frequency shift in a cavity's resonant frequency can be induced by various causes, including fluctuations in the helium gas pressure or the influence of mechanical vibrations. Such frequency shifts should be controlled by the piezo tuner by applying a static piezo voltage. The adjustment of a frequency shift requires a high reproducibility of the frequency change by the piezo tuner. Measurements of the frequency changes made by applying a repeated step voltage to the piezo tuner (Fig. 14, left) were carried out. The reproducibility results are shown at the right in Fig. 14. The frequency change during ten cycles was not constant, and relatively large similar fluctuations in the resonant frequency were observed in all four cavities. The friction at the supporting tabs for the He jacket or at the supporting rods for the tuner bellows, produced by driving the tuner system, is supposed to be one potential cause. A way to decrease the friction from driving the tuner system is needed to improve the reproducibility.

\section{Higher order modes}

Higher order mode (HOM) couplers are used for extracting the harmful higher order modes induced by a beam 

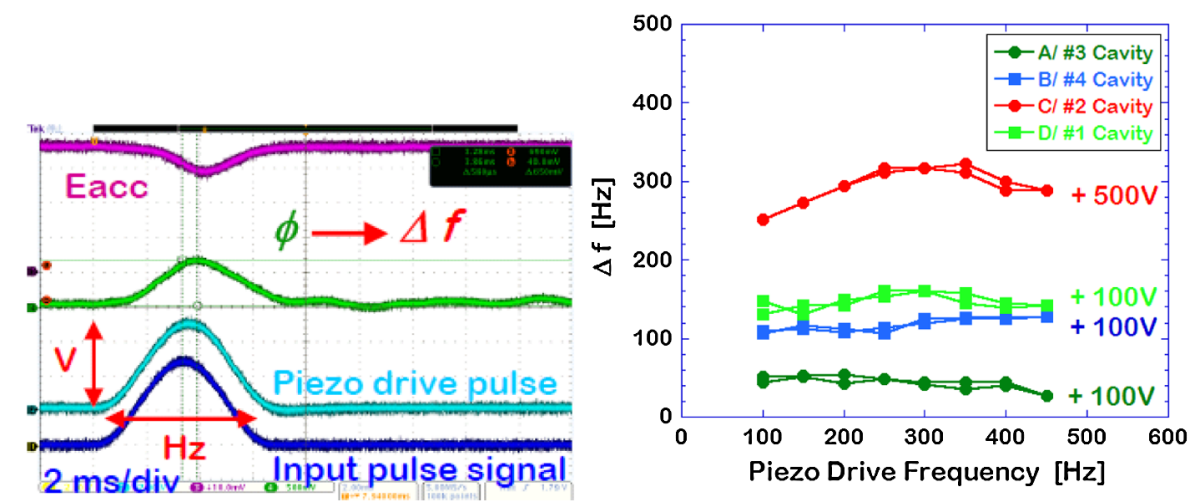

FIG. 13. (Color) Pulse response signals driven by piezo tuner with single pulse (left) and frequency change $(\Delta f)$ as a function of drive frequency with piezo tuner (right).

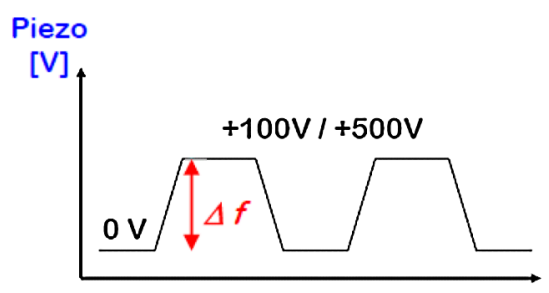

Time $[\mathrm{min}]$

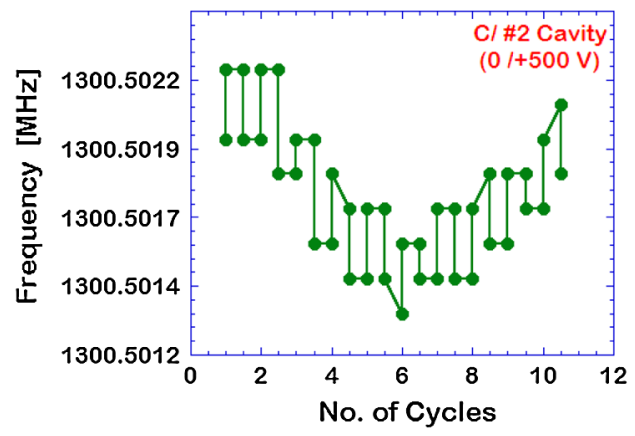

FIG. 14. (Color) Measurements of reproducibility of frequency changes by applying repeated step voltage to piezo tuner: The left figure shows an applied piezo voltage with a repeated step pattern (one cycle represents approximately 10 min.). The right figure shows the change in the resonant frequency from applying a piezo voltage between 0 and $+500 \mathrm{~V}$ in \#2 cavity.

passing through a superconducting cavity. The HOM coupler has a loop antenna made of niobium to couple with both electric fields and magnetic fields, as shown in Fig. 4.

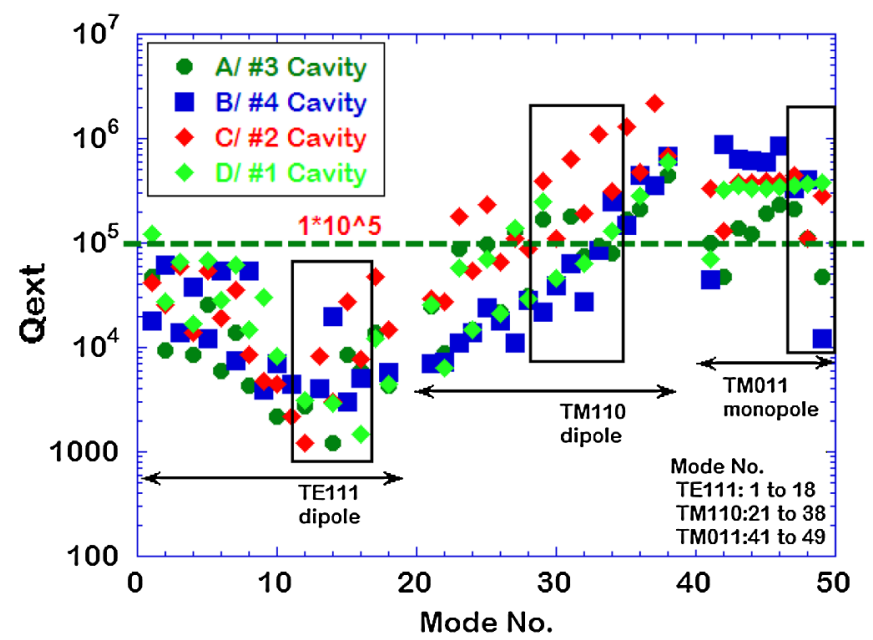

FIG. 15. (Color) External $Q$ values $\left(Q_{\text {ext }}\right)$ of higher order modes in four Tesla-like cavities. The $Q_{\text {ext }}$ is the total value in the HOM-1 and HOM-2 couplers located on both sides of the cavity. The black squares show that the dangerous higher order modes with high impedance have strong coupling with a beam.
Since it is possible for HOM couplers to couple with an accelerating mode, in addition to higher order modes, the HOM couplers have wideband rf characteristics with a notch filter to reject the accelerating mode. The measured external $Q$ values $\left(Q_{\text {ext }}\right)$ of the higher order modes in the four Tesla-like cavities are shown in Fig. 15. Although the $Q_{\text {ext }}$ requirement for suppressing the beam instability is estimated to be less than $10^{5}$ [5], several modes with $Q_{\text {ext }}$ values of higher than $10^{5}$ were found in some of the TM110 dipole modes and TM011 monopole modes. Adjusting both the direction of the loop antenna to the beam axis and the location of the antenna tip is considered to be effective to obtain sufficient coupling with these modes and reduce the $Q_{\text {ext }}$ values.

\section{Mechanical vibration modes}

It is useful to use a piezo element as a sensor to detect mechanical vibrations. The output signal from a piezo sensor is shown in Fig. 16. The mechanical vibrations excited by an rf pulse with a flattop pulse width of $1 \mathrm{~ms}$ and a repetition rate of $5 \mathrm{~Hz}$ were gradually damped with time, and the amplitude was sufficiently reduced to less than $1 / 10$ at the beginning of the next pulse. Therefore, mechanical vibrations do not seem to pose a problem for stable pulsed operation at $5 \mathrm{~Hz}$. However, the damping of 

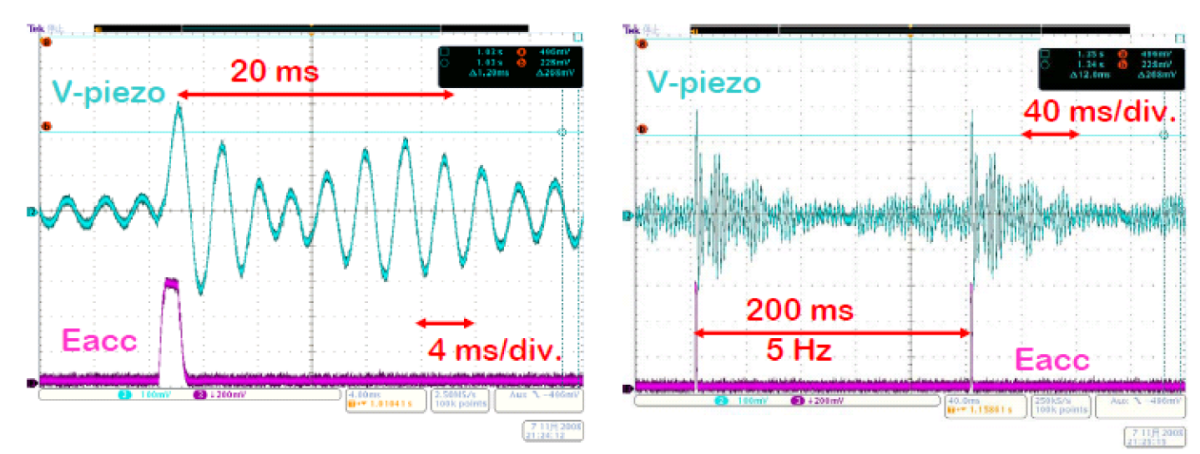

FIG. 16. (Color) Observation of mechanical vibrations during pulsed operation at $5 \mathrm{~Hz}$ and $28.2 \mathrm{MV} / \mathrm{m}$ in $\mathrm{C} / \# 2$ cavity. The light blue line is the output voltage from the piezo sensor. The purple line is an acceleration gradient operated with a pulsed mode. The left image has a shorter time range with $4 \mathrm{~ms}$ per division, and the right has a longer time range with $40 \mathrm{~ms}$ per division.

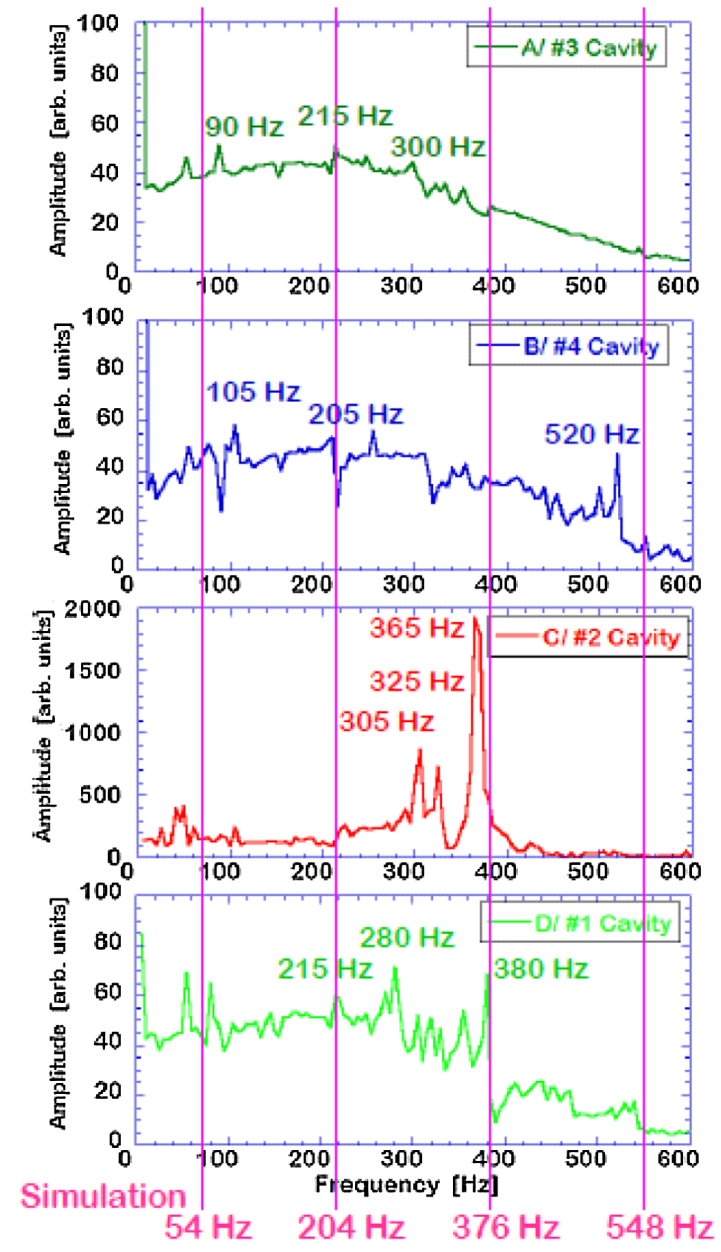

FIG. 17. (Color) FFT analysis of mechanical vibration modes in four cavities. The mechanical vibration modes between 0 and $600 \mathrm{~Hz}$ are shown, and no remarkable mode above $600 \mathrm{~Hz}$ is observed. The accelerating gradients in each cavity are $18.4 \mathrm{MV} / \mathrm{m}$ (\#3 cavity), $18.6 \mathrm{MV} / \mathrm{m}$ (\#4 cavity), $28.2 \mathrm{MV} / \mathrm{m}$ (\#2 cavity), and 18.0 MV/m (\#1 cavity). The purple lines show the mechanical vibration modes obtained by the simulation in the model calculations. The different vertical scale in the figures is due to the sensitivity of the different piezo elements used in the four cavities; low-voltage-type piezos (\#3, \#4, and \#1 cavity) and high-voltage-type piezo (\#2 cavity). mechanical vibration modes after 20 ms was still insufficient, so that a severe problem would be expected in case of a higher repetition rate like $50 \mathrm{~Hz}$.

The mechanical vibrations in the four Tesla-like cavities were measured and found to be similar to Fig. 16, and the mechanical vibration modes were analyzed by fastFourier-transform (FFT), as shown in Fig. 17. In the model calculation, mechanical vibration modes were obtained with frequencies of $54 \mathrm{~Hz}$ ( 0 order), $204 \mathrm{~Hz}$ (1st order), $376 \mathrm{~Hz}$ (3rd order), and $548 \mathrm{~Hz}$ (4th order), as longitudinal vibration modes which the whole cell structure of the ninecell cavity shifts in parallel to the beam axis. The calculated mechanical vibration modes were found in the components of the measured mechanical vibration modes, but the frequency of the dominant mode was different in each cavity. Further investigation is needed to determine the reason.

\section{HIGH FIELD PERFORMANCE}

One of the four cavities achieved a stable pulsed operation at $32 \mathrm{MV} / \mathrm{m}$, which is higher than the operating accelerating gradient in the ILC. The typical pulse signals with and without an rf feedback control are shown in Fig. 18. The input rf power of $340 \mathrm{~kW}$ was reduced to $240 \mathrm{~kW}(70 \%)$ after $0.5 \mathrm{~ms}$ by a step pulse. Although cavity phase change and accelerating gradient decrease are seen in the left figure, they were precisely maintained constants by the rf feedback control in the right figure [10]. A comparison of the maximum acceleration gradient $\left(E_{\text {acc, max }}\right)$ obtained in the cryomodule tests with that in the vertical tests is shown in Fig. 19 . The average $E_{\text {acc, max }}$ values in the vertical and cryomodule tests $(1.5 \mathrm{~ms})$ were 22.7 and $23.7 \mathrm{MV} / \mathrm{m}$, respectively. Therefore, it is concluded that the cavity performance was maintained in the cryomodule with no severe degradation. Since the average $E_{\text {acc, max }}$ in the shorter pulse width of $0.6 \mathrm{~ms}(0.1 \mathrm{~ms}$ at the flattop) was $28.3 \mathrm{MV} / \mathrm{m}$, it is considered that the achievable $E_{\text {acc,max }}$ might be limited by the total amount of heat loss at the hot spot. 


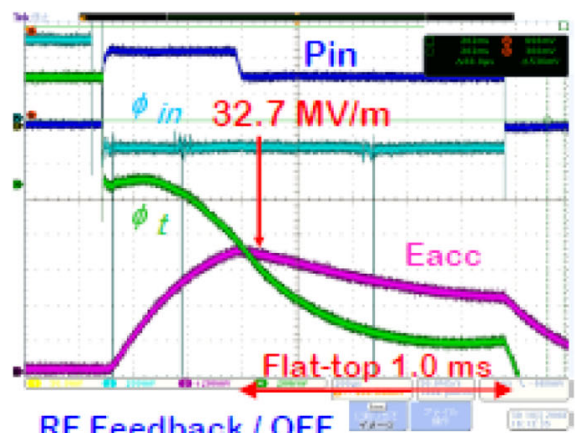

RF Feedback / OFF

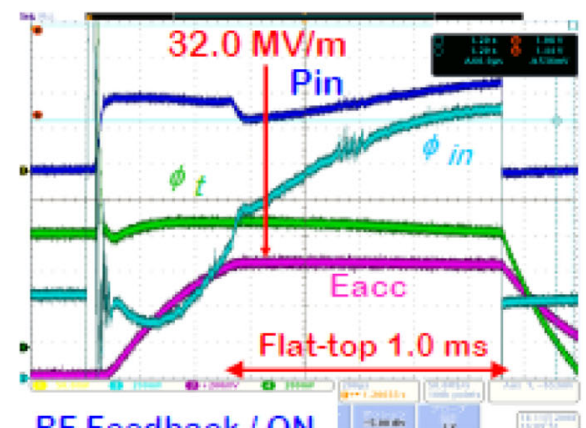

RF Feedback/ON

FIG. 18. (Color) Pulsed signals at high field operation in C/\#2 cavity without an rf feedback control (left) and with rf feedback control (right). The light blue line is the input phase $\left(\phi_{\text {in }}\right)$ between the input rf power and a reference. The green line is the cavity phase $\left(\phi_{t}\right)$ between the rf power transmitted through a cavity and a reference. The dark blue line is the input rf power, and the purple line is the accelerating gradient.

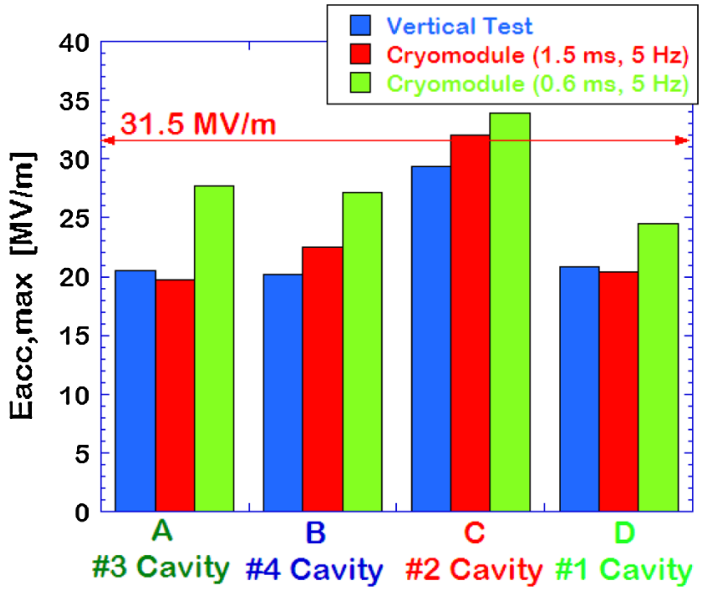

FIG. 19. (Color) Comparison between $E_{\text {acc, max }}$ values of vertical $\mathrm{cw}$ tests and cryomodule tests in pulsed operation with $1.5 \mathrm{~ms} / 0.6 \mathrm{~ms}$ and $5 \mathrm{~Hz}$.

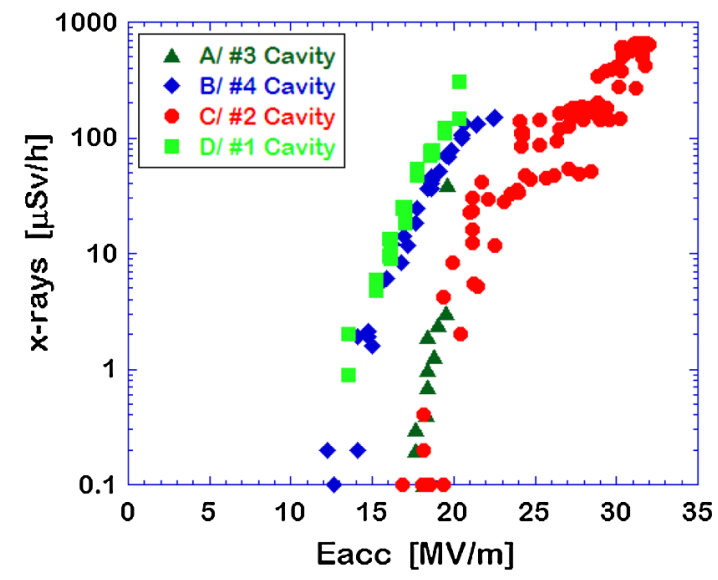

FIG. 20. (Color) Relation between x-ray radiation levels and accelerating gradients in four cavities in pulsed operation with $1.5 \mathrm{~ms}$ and $5 \mathrm{~Hz}$.
The observations of the $\mathrm{x}$-ray radiation in each cavity are shown in Fig. 20. Heavy x-ray radiation due to field emission higher than $100 \mu \mathrm{Sv} / \mathrm{h}$ was detected by a sensor located just under the cryomodule. Although the tolerable radiation limit is presently not clear, the radiation level due to field emission should be suppressed as low as possible. Contamination by dust particles during the cavity string assembly in the clean room or during the beam-tube connection in the STF tunnel might potentially be considered as one of the causes.

\section{LORENTZ FORCE DETUNING}

The resonant frequency of a cavity gradually decreases due to the deformation of the cell shape by Lorentz force during an $\mathrm{rf}$ pulse. Even if the detuning effect can be precisely compensated by a vector-sum feedback control without a piezo drive pulse, very large input rf power is required to maintain the constant high accelerating gradient. It is very important that the detuning frequency should be compensated by a piezo tuner as small as possible in order to minimize the required rf power. Since the cavity phase $\left(\phi_{t}\right)$ of the rf power transmitted through a cavity changes exponentially after the end of an rf pulse (see Figs. 18 and 23), the cavity detuning frequency $(\Delta f)$ can be obtained by the time constant of the decay time: $\Delta f=(1 / 2 \pi) \cdot d \phi_{t} / d t$. Figure 21 shows an example of Lorentz force detuning at $30 \mathrm{MV} / \mathrm{m}$ in a stable pulsed operation obtained by a pulse-cut method [10], in which the pulse width was changed from 0.1 to $1.5 \mathrm{~ms}$ in $0.1 \mathrm{~ms}$ increments. The measured detuning frequencies $(\Delta f)$ during the $0.5 \mathrm{~ms}$ rise time and at the $1.0 \mathrm{~ms}$ flattop was -360 and $-315 \mathrm{~Hz}$, respectively. The detuning frequency in the rise time can be compensated by predetuning or offset detuning, which involves setting the resonant frequency of a cavity higher than the drive frequency of the rf power source in advance (see Fig. 24). It is necessary to compensate for the detuning frequency at the flattop by using a piezo tuner. Figure 22 shows a detuning frequency comparison between three Tesla cavities with a Saclay tuner 


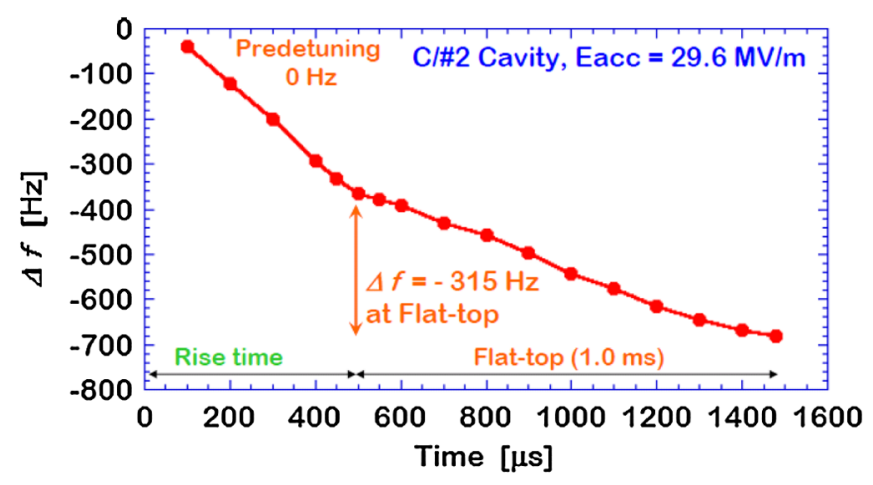

FIG. 21. (Color) Observation of Lorentz-detuning frequency $(\Delta f)$ at $30 \mathrm{MV} / \mathrm{m}$ measured by pulse-cut method; rf feedback control: ON, predetuning: $0 \mathrm{~Hz}$, piezo tuner: OFF.

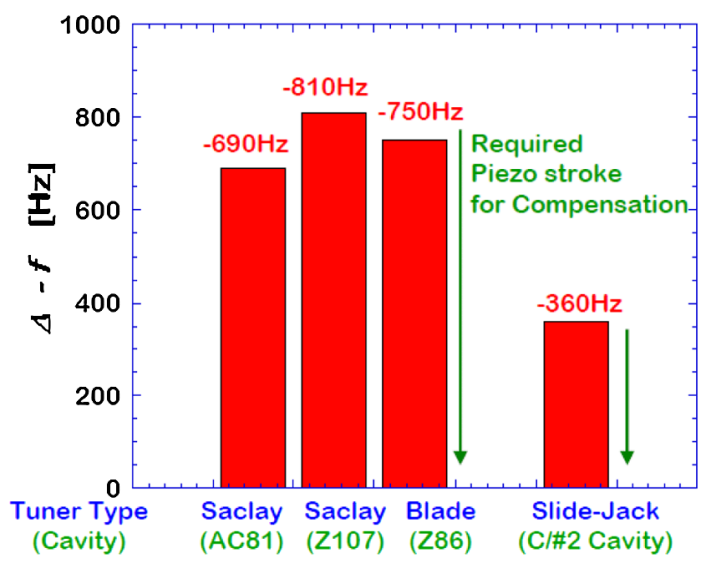

FIG. 22. (Color) Comparison between Lorentz-detuning frequencies $(\Delta-f)$ of different cavities with different tuner systems under the normalized operational condition with $E_{\text {acc }}$ of $31.5 \mathrm{MV} / \mathrm{m}$ and flattop pulse width of $1.0 \mathrm{~ms}$.

[11] or blade tuner [12] and the Tesla-like cavity with a slide-jack tuner under the normalized condition for the operational gradient at $31.5 \mathrm{MV} / \mathrm{m}$ and flattop pulse width of $1 \mathrm{~ms}$. The detuning frequency of $-360 \mathrm{~Hz}$ at
$31.5 \mathrm{MV} / \mathrm{m}$ (calculated from $-315 \mathrm{~Hz}$ at $30 \mathrm{MV} / \mathrm{m}$ by $\Delta f=$ Const $\left.\cdot[\text { Eacc }]^{2}\right)$ in the Tesla-like cavity $(\mathrm{C} / \# 2$ cavity) was much lower than that in three Tesla cavities tested at DESY; the estimated detuning frequencies at $31.5 \mathrm{MV} / \mathrm{m}$ and $1 \mathrm{~ms}$ flattop are $-690 \mathrm{~Hz}$ in the AC81 cavity with the Saclay tuner [11], $-810 \mathrm{~Hz}$ in the Z107 cavity with the Saclay tuner [13], and $-750 \mathrm{~Hz}$ in the Z86 cavity with the blade tuner [12]. This was the result of the improved stiffness of the Tesla-like cavity. This effect was helpful for reducing the required piezo stroke and minimizes the tuning deviation at the flattop by compensation. It can be concluded that the effectiveness of the stiff cavity structure in the Tesla-like cavity was clearly confirmed in this experiment.

Compensation of the Lorentz force detuning was carried out based on the optimized parameters obtained in the low power measurements of the piezo tuner. An applied voltage of $+500 \mathrm{~V}$, frequency of $300 \mathrm{~Hz}$, and delay time of $0.6 \mathrm{~ms}$ from the piezo input pulse to the rf pulse were set as the parameters of the piezo drive pulse, as shown in Fig. 23. In the case of the application with the piezo drive pulse (right), the input rf power signal at the flattop was kept constant due to the efficient compensation of the detuning frequency by the piezo tuner. On the other hand, in the case of no piezo drive (left), the input rf power signal at the flattop was gradually increased in order to maintain a constant Eacc value in spite of the frequency shift by Lorentz detuning. A series of successful compensations of Lorentz force detuning at $31 \mathrm{MV} / \mathrm{m}$ by the predetuning and piezo tuner is shown in Fig. 24. The predetuning was adjusted to $+360 \mathrm{~Hz}$, and the parameters of the piezo drive pulse were optimized for the drive frequencies of 250, 300, 350 , and $400 \mathrm{~Hz}$ with a suitable delay time between the piezo drive pulse and an rf pulse at the fixed applied voltage of $+500 \mathrm{~V}$. A tuning deviation of less than $\pm 50 \mathrm{~Hz}$ at the flattop was achieved in a wide parameter range for driving the piezo tuner. A tuning deviation of $\pm 50 \mathrm{~Hz}$ is the target value in the ILC, and this criteria was determined by the limitation of a margin of $10 \%$ in avail-
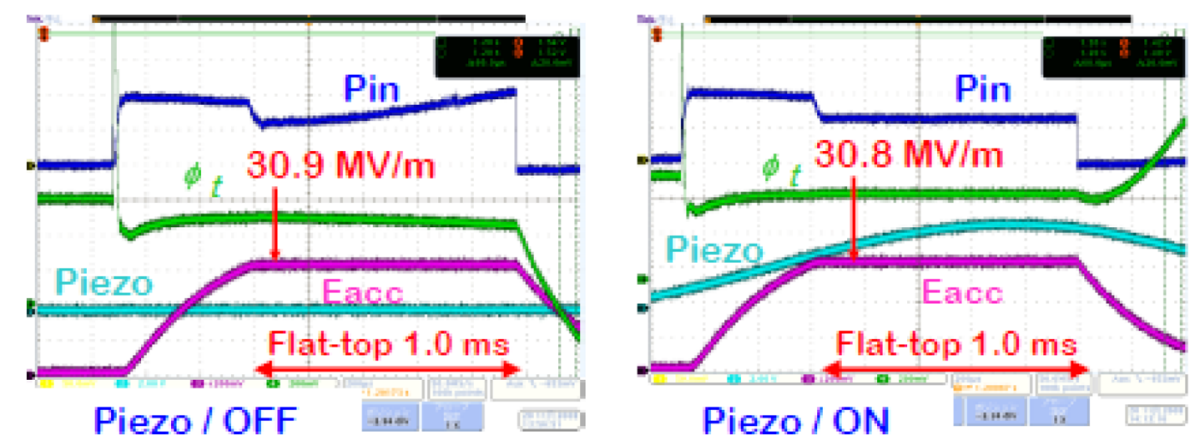

FIG. 23. (Color) Pulsed signals at high field operation in C/\#2 cavity without piezo drive voltage (left) and with piezo drive voltage (right). The light blue line is the piezo drive voltage. The green line is the cavity phase $\left(\phi_{t}\right)$ between the transmitted rf power through a cavity and a reference. The dark blue line is the input $\mathrm{rf}$ power, and the purple line is the accelerating gradient. The accelerating gradient (pink line) and cavity phase (green line) were kept constant by an rf feedback control. The obtained amplitude and phase stabilities were $0.01 \% \mathrm{rms}$ and $0.02 \mathrm{deg}$ rms by the rf feedback control system [10]. 


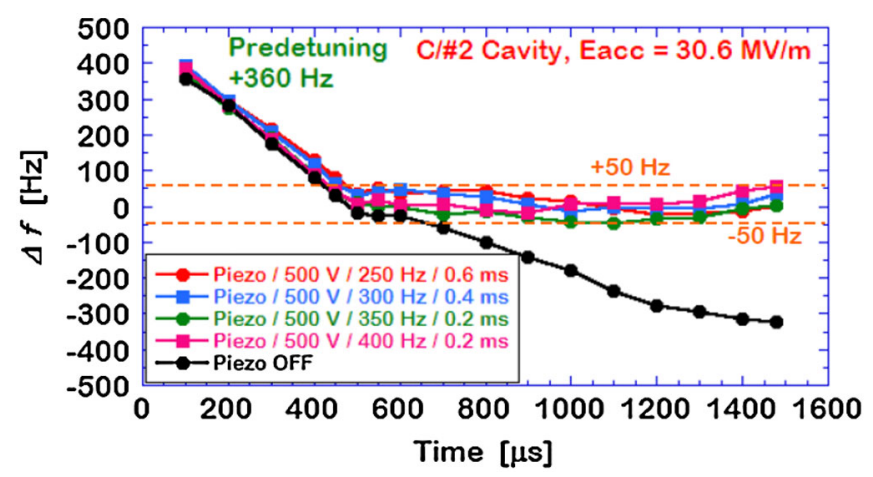

FIG. 24. (Color) Successful compensation of Lorentz force detuning by detuning and piezo tuner. Several optimized parameters for the frequency and delay time were found at the fixed applied voltage of $+500 \mathrm{~V}$ for the piezo drive pulse. The data in the case of no piezo drive (black dots) are added for comparison. (Detailed results are also found in [17].)

able input rf power. The corresponding phase value $(\psi)$ is \pm 13 degrees in $Q_{L}=3.0 \times 10^{6}$ by a calculation with $\tan (\psi)=-2 \cdot Q_{L} \cdot(\Delta f / f)$. Here, compensation of Lorentz force detuning was successfully demonstrated at $31 \mathrm{MV} / \mathrm{m}$ in the Tesla-like cavity by detuning and the piezo tuner.

\section{DYNAMIC LOSS MEASUREMENT}

The simultaneous operation of four Tesla-like cavities was carried out with a vector-sum feedback control [10] in the high power rf distribution system, as shown at the right in Fig. 9. Figure 25 shows the accelerating gradients and cavity phases of the four cavities. A predetuning of $100-150 \mathrm{~Hz}$ was adjusted in each cavity, and no piezo drive pulse was applied. The sum of the four accelerating gradients was kept constant at the flattop, and an average accelerating gradient of $17.2 \mathrm{MV} / \mathrm{m}$ and total accelerating voltage of $71 \mathrm{MV}$ were achieved. The deviation in the cavity phase was less than \pm 5 degrees at flattop, and stable operation was confirmed for a long operation time of several hours. The phase deviation of \pm 5 degrees in $Q_{L}=$ $2.0 \times 10^{6}$ in this case is corresponding to the frequency deviation of approximately $\pm 30 \mathrm{~Hz}$. Increment of input $\mathrm{rf}$ power compensated by a vector-sum control is $1.5 \%$ in this experiment without a piezo drive pulse. In order to efficiently increase the total accelerating voltage of the four cavities, a precise rf power dividing system is required that is suitable to achieve the $E_{\text {acc,max }}$ of each cavity.

The dynamic rf loss was measured at $2 \mathrm{~K}$ by the flow rate of the evaporated helium gas in the cryomodule [3]. The total rf loss of the four Tesla-like cavities operated with the vector-sum control of the resonance is shown as a function of the average input power to the four cavities in Fig. 26, together with the total heat load at the four input couplers (off resonance). The difference between these two values represents the net rf loss at the cavity's inner surface. The measured heat load at $2 \mathrm{~K}$ at the input couplers was much higher than the calculated value of $0.03 \mathrm{~W}$ per coupler at an input power of $350 \mathrm{~kW}$.

The rf loss at a cavity's inner surface gives a $Q_{o}$ value, as shown in Fig. 27. Although the $Q_{o}$ values dropped due to field emission (see Fig. 20), $Q_{o}$ values higher than $10^{10}$ at the lower fields are shown in the figure. This result at the lower fields indicates the good shielding effect of the magnetic shield inserted in the helium vessel, as explained in Fig. 6.

\section{FUTURE PROJECTS}

The next STF project at KEK is called the S1-Global [14], which will employ a cryomodule containing four Tesla-like cavities from KEK, two Tesla cavities from DESY, and two Tesla cavities from FNAL, in an international collaboration for the ILC. The string assembly of these eight cavities will begin in January 2010, and high power tests of the cryomodule at $2 \mathrm{~K}$ are scheduled from September to December 2010. Vertical tests of the Teslalike cavities for the S1-Global have continued until November 2009, and the average $E_{\text {acc,max }}$ obtained has
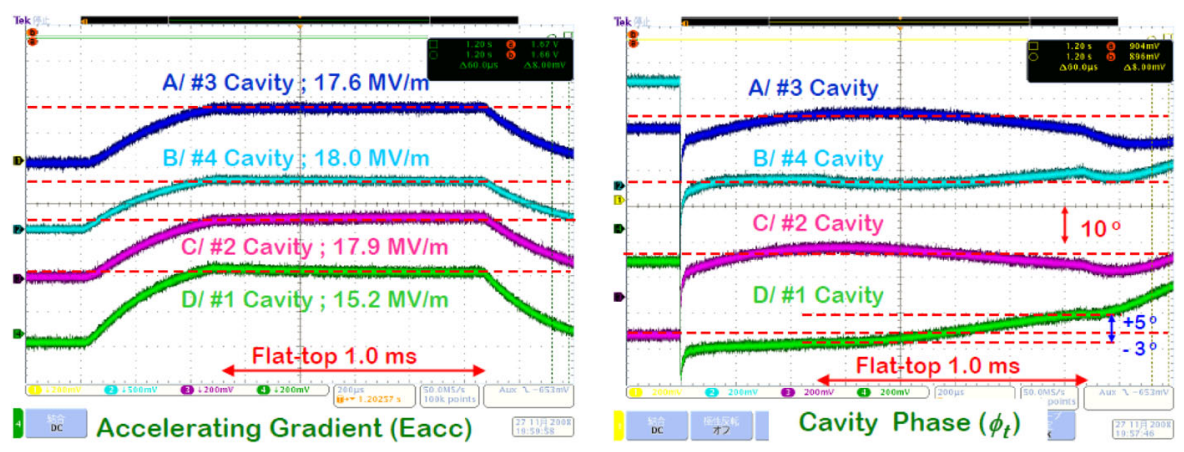

FIG. 25. (Color) The rf pulse signals of accelerating gradient (left) and cavity phases (right) in four-cavity operation with vector-sum control without a piezo drive pulse. Red broken lines show the constant accelerating gradient at flattop (left) and datum line of the standard cavity phase (right). 


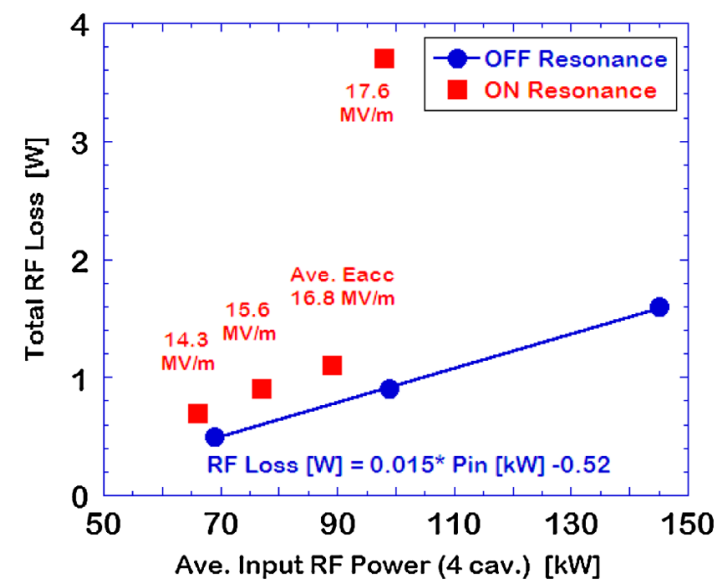

FIG. 26. (Color) Measurement of total dynamic rf losses at $2 \mathrm{~K}$ in four-cavity simultaneous operation with off-resonance (blue) and on-resonance (red). The measured static loss of $9 \mathrm{~W}$ has already been subtracted in this figure.

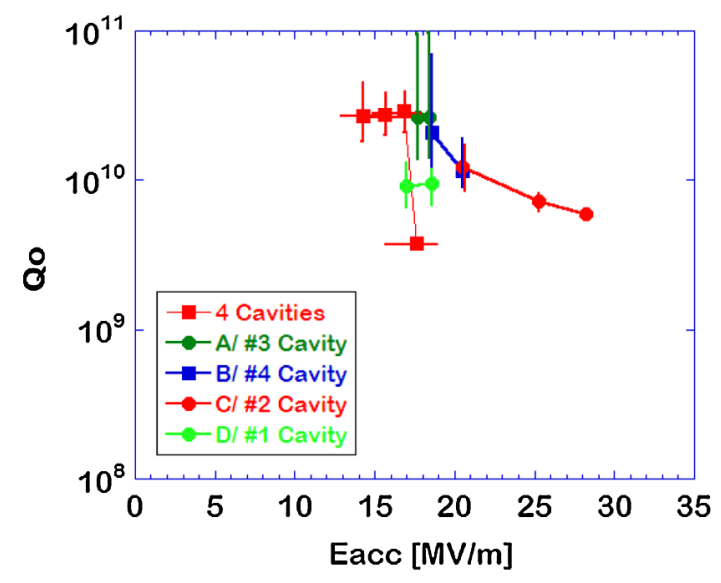

FIG. 27. (Color) Unloaded $Q$ values $\left(Q_{o}\right)$ calculated from the results of dynamic rf loss measurement at $2 \mathrm{~K}$.

increased to $28 \mathrm{MV} / \mathrm{m}$ as a result of several improvements in both the cavity fabrication techniques and the surface preparation methods at KEK. Efforts will continue to achieve the target values for the ILC.

After the S1-Global, a capture cryomodule consisting of two Tesla-like cavities will be installed in the STF tunnel in 2011 for the Quantum-Beam project [15] with a beam operation. After this, the first cryomodule to include nine Tesla-like cavities for the STF phase-II project [16] will be completed in 2012. The fabrication of a total of 11 Teslalike cavities for these projects at KEK started in 2009. One rf unit consisting of three cryomodules driven by a 10-MW klystron is the final step planned as a part of the STF phaseII project. Research and development of the superconducting cavity system for the STF phase-II has been continuing, with the aim to realize the construction of the future ILC.

\section{CONCLUSIONS}

The cryomodule tests in the STF phase-I project were finished by the end of December 2008, after approximately four months of operation at $2 \mathrm{~K}$. Low and high rf power tests of the STF cryomodule containing four Tesla-like nine-cell cavities were successfully carried out without any serious problems. Several remarkable results were obtained through the STF phase-I experiments, and these are summarized as follows: (i) No severe $E_{\text {acc, max }}$ degradation in comparison with the vertical test results was observed in any of the four cavities. (ii) A stable pulsed operation at $32 \mathrm{MV} / \mathrm{m}$ was confirmed in one of the four cavities. This value exceeds the target for the operational accelerating gradient in the ILC. (iii) The effectiveness of the stiff support structure in the Tesla-like cavities was clearly shown in the reduced Lorentz-detuning frequency at the flattop in the pulsed operation. (iv) Successful compensation of the Lorentz force detuning at $31 \mathrm{MV} / \mathrm{m}$ was demonstrated by predetuning and a piezo tuner. The tuning deviation was less than $\pm 50 \mathrm{~Hz}$ at the flattop, which is the target value for the ILC. (v) Although the average operating gradient was lower than the target value in ILC, the stable performance as a total superconducting cavity system including couplers and tuners was realized as the first step in the four-cavity operation with the vector-sum control by one klystron.

On the other hand, several improvements mentioned below will be required for the next step.

(i) It is necessary to reduce the friction at the supporting tabs for the He jacket and at the tuner mechanism in order to control the piezo tuner more precisely and more reproducibly.

(ii) It is important to suppress the $\mathrm{x}$-ray radiation level due to field emitted electrons by more careful assembly work and maintaining a cleaner environment during the assembly of the four cavities and the installation of the beam tubes.

(iii) It is necessary to eliminate the discharge phenomenon in the high power rf distribution system, which includes the input couplers, circulators, power dividers, dummy loads, and waveguide system.

(iv) The static and dynamic heat losses from the input couplers should be reduced.

(v) The piezo element type should be correctly selected to obtain a sufficient stroke at low temperature.

Implementing these improvements would enhance the performance in the next superconducting cavity system.

\section{ACKNOWLEDGMENTS}

The authors would like to thank the members of the STF project at KEK, consisting of the LLRF group, HLRF group, cryomodule group, cryogenics group, and linear collider office. Special thanks are due to the staff of Mitsubishi Heavy Industries, Ltd. for manufacturing the 
cavities and Hitachi, Ltd. for fabricating the 6-m cryomodule.

[1] S. Noguchi, in SRF'05 (Cornell University, Ithaca, 2005), http://www.Ins.cornell.edu/public/SRF2005/ in the ILC seminar at KEK, 2007, http://lcdev.kek.jp/ LocalMeetings/.

[2] E. Kako et al., in Proceedings of the 2007 Particle Accelerator Conference, Albuquerque, New Mexico (IEEE, Albuquerque, New Mexico, 2007), pp. 2107-2109.

[3] N. Ohuchi et al., in Proceedings of the 11th European Particle Accelerator Conference, Genoa, 2008 (EPS-AG, Genoa, Italy, 2008), pp. 892-894.

[4] E. Kako et al., Proceedings of the 11th European Particle Accelerator Conference, Genoa, 2008 (Ref. [3]), pp. 868870.

[5] B. Aune et al., Phys. Rev. ST Accel. Beams 3, 092001 (2000).

[6] S. Noguchi, E. Kako, and K. Kubo, in Proceedings of the 4th Workshop on RF Superconductivity (KEK, Tsukuba,
Japan, 1989), pp. 397-412.

[7] K. Watanabe et al., Nucl. Instrum. Methods Phys. Res., Sect. A 595, 299 (2008).

[8] E. Kako et al., in SRF'07 (Peking University, Beijing, China, 2007), WEP10.

[9] S. Fukuda et al., in Proceedings of the 2009 Particle Accelerator Conference, Vancouver, BC (IEEE, New York, 2009), TU5PFP086.

[10] S. Michizono et al., Proceedings of the 2009 Particle Accelerator Conference, Vancouver, BC (Ref. [9]), WE5PFP083.

[11] L. Lilje, SRF'07 (Ref. [8]), MO102.

[12] C. Pagani et al., SRF'07 (Ref. [8]), TUP72.

[13] L. Lilje (private communication).

[14] H. Hayano, SRF'09 (Helmholtz Zentrum, Berlin, Germany, 2009), MOODAU02.

[15] J. Urakawa (private communication).

[16] K. Yokoya, SRF'09 (Ref. [14]), FROBAU07.

[17] Y. Yamamoto et al., Proceedings of the 2009 Particle Accelerator Conference, Vancouver, BC (Ref. [9]), TU5PFP075. 